\title{
Improving the Timed Automata approach to biological pathway dynamics
}

\author{
Rom Langerak, Jaco van de Pol, Janine N. Post, and Stefano Schivo \\ University of Twente, Enschede, The Netherlands
}

\begin{abstract}
Biological systems such as regulatory or gene networks can be seen as a particular type of distributed systems, and for this reason they can be modeled within the Timed Automata paradigm, which was developed in the computer science context. However, tools designed to model distributed systems often require a computer science background, making their use less attractive for biologists. ANIMO (Analysis of Networks with Interactive MOdeling) was built with the aim to provide biologists with access to the powerful modeling formalism of Timed Automata in a user friendly way. Continuous dynamics is handled by discrete approximations.

In this paper we introduce an improved modeling approach that allows us to considerably increase ANIMO's performances, opening the way for the analysis of bigger models. Moreover, this improvement makes the introduction of model checking in ANIMO a realistic feature, allowing for reduced computation times. The user interface of ANIMO allows to rapidly build non-trivial models and check them against properties formulated in a human-readable language, making modeling a powerful support for biological research.
\end{abstract}

\section{Introduction}

To understand the possible causes of a disease and design effective cures it is necessary to closely study the behavior exhibited by biological cells under particular conditions. A signaling pathway describes the chain of interactions occurring between the reception of a signal and the response with which the cell reacts to such signal. A signal is typically represented by a substance which can bind to specific receptors on the cell surface, activating them. Active molecules relay the signal inside the cell by activating other molecules until a target is reached. The target of a signaling pathway is usually a transcription factor, a molecule with the task of controlling the production of some protein. Such regulation is considered to be the response of the cell to the received signal.

The current knowledge on signaling pathways (mostly organized in databases such as KEGG [11] or PhosphoSite [10]) suggests that the interactions involved in a cellular response assume more often the shape of a network than that of a simple chain of signal relays. Such networks are typically highly connected, involving feedback loops and crosstalk between multiple pathways, making it 
difficult to grasp their dynamic behavior. For this reason, computational support is required when studying non-trivial biological networks.

A number of software tools are available for modeling complex networks of biochemical interactions $[3,9,14,24,8]$. These tools significantly contribute to the process of formalizing the knowledge on biological processes, rendering them amenable to computational analysis. However, a lack of familiarity with the formalisms underlying many available tools hampers their direct application by biology experts. ANIMO (Analysis of Networks with Interactive MOdeling, [2, 19, 22, 18]) is a software tool based on the formalism of Timed Automata [1] that supports the modeling of biological signaling pathways by adding a dynamic component to traditional static representations of signaling networks. ANIMO allows to compare the behavior of a model with wet-lab data, and to explore such behavior in a user-friendly way. In order to achieve a good level of user-friendliness for a public of biologists, the complexity of Timed Automata is hidden under the hood, presenting ANIMO as an app for Cytoscape [12], a tool specifically developed for visualizing and elaborating biological networks. Additionally, a web interface for ANIMO has been developed [23], that allows to access the tool from any web browser without the need to install any software. Students in biology and bio-medical courses have been using ANIMO to learn about signaling networks, explain existing data and plan experiments. Thanks to the students' feedback the features of ANIMO have been constantly improving. Moreover, the tool is currently being applied in biological research to gain insight on cell differentiation. Models are built and managed by biologists independently, and provide a useful and visually appealing way to represent the key interactions under investigation.

Previously, ANIMO supported only interactive exploration of network dynamics based on simulation runs. Model checking queries could be answered through the UPPAAL tool [13], but the required knowledge of temporal logic together with the usually long response times slowed down the investigation process. In order to encourage the use of model checking on non-trivial models of signaling networks, we updated ANIMO with a new way of modeling reactions. This marks a relevant improvement in terms of performance with respect to the model previously used in ANIMO. Moreover, consistently with the intents of our tool, we implemented also a user interface for the definition of model checking queries in ANIMO. This allows a user to interrogate an ANIMO model without requiring previous experience in temporal logics. These new features are available in the latest version of ANIMO, which was recently reimplemented as a Cytoscape 3 app [4]: this lets users profit from the additional analysis features made available by the other apps in the Cytoscape environment.

The paper continues as follows. After introducing the basic concepts in Section 2, we illustrate in Section 3 a new way of using Timed Automata in ANIMO. In Section 4 we present a comparison between the new modeling approach and the one previously used in ANIMO, focusing on model analysis performances. In Section 5 we describe how model checking was made accessible to ANIMO users. We conclude the paper with Section 6, discussing future work. 


\section{Preliminaries}

\subsection{Signaling pathways in biology}

A signaling pathway is an abstract representation of the reactions occurring inside a biological cell when, e.g., a signaling substance comes in contact with the cell surface receptors. In this setting, a reaction is the interaction between two components: the upstream enzyme (the molecule holding the active role in the reaction) and the downstream substrate (the passive molecule). The enzyme can be for example a kinase, which attaches a phosphate group to its substrate, performing a phosphorylation: this determines a change in the shape of the substrate and consequently a new function (Fig. 1). The new state reached by the substrate is often called active: if the substrate of the reaction is itself a kinase, it can then proceed in passing on the signal by activating its own target molecule, continuing a chain of reactions leading to the target of the signaling chain. Such target is usually a transcription factor, i.e. a molecule that influences the genetic response of the cell, for example promoting the production of a particular protein.

Pathways are traditionally represented in a nodes-edges form (see Fig. 1a), with nodes representing molecular species and edges standing for reactions, where $\rightarrow$ represents activation and $\dashv$ represents inhibition (i.e. inactivation).

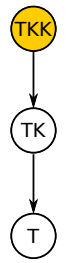

(a)

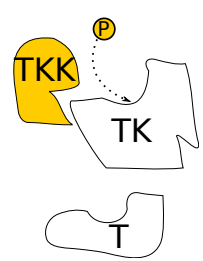

(b)

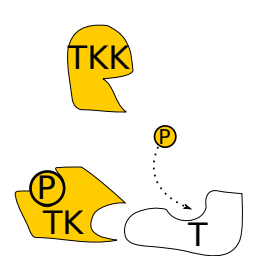

(c)

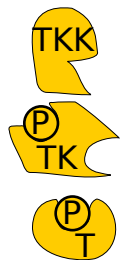

(d)

Fig. 1. A kinase signaling network represented in the nodes-edges form (a) and its evolution represented by abstract molecular interactions (b-d). T is the target of the signaling pathway, TK is the kinase that activates T, and TKK is the kinase that activates TK. (b) An already active (yellow) TKK can bind to an inactive (empty) TK, catalyzing its phosphorylation (i.e. binding of a phosphate group, P). This causes a change in the shape of TK, activating its enzymatic function (c). The active TK can in turn activate its target $T$, enabling it to carry out its function (d).

The current knowledge on signaling pathways $[11,10]$ evidences the fact that signaling interactions are rarely a simple chain of activations as represented in Figure 1a. More often, they assume the shape of a network with multiple feedback loops and crosstalk from different signaling sources. This complexity is an added difficulty for the study of such networks, reducing the possibilities to deduce the dynamic behavior of a signaling network by inspecting its static representation. For this reason, efficient computational support is essential when representing and analyzing the behavior of complex signaling networks. 


\subsection{Timed Automata}

Timed Automata (TA) are finite-state automata enriched with real-valued clocks and synchronization channels. All clocks in a TA system advance with the same rate, and transitions between the locations of an automaton depend on conditions on clocks. In particular, a guard defines when a transition may be taken, while an invariant is the condition for permanence in a location. A transition can also allow two automata to synchronize, with each participant performing one of two complementary actions (input and output) on a synchronization channel. A set of clocks may also be reset by a transition, causing them to restart from 0 .

The models we will present here were implemented using the software tool UPPAAL [13], which adds a number of features to the basic definition of TA. Some of these extensions include: support for integer variables in addition to clocks, broadcast synchronization channels (one sender, many receivers), definition of C-like functions to perform more operations besides clock resets. UPPAAL also allows for a special type of locations, named committed (marked with a $C$ in the graphical representation). As long as an automaton is in a committed location, time is not allowed to flow. This feature can be used for example to perform immediate updates to local variables before letting the computation proceed. Examples of the listed features are found in the TA model in Section 3.2.

\subsection{Activity-based models in ANIMO}

ANIMO allows the definition of activity-based models. This means that we assume each signaling molecule in a cell to be at any time in one of two states: active or inactive. Active molecules can take an active role in reactions, changing the state of other molecules, activating inactive molecules or inhibiting (i.e. deactivating) active molecules. In a kinase-based signaling network an activation process can be a phosphorylation, and it is usually countered by the corresponding dephosphorylation. However, our models are not limited to kinase networks: other features like different post-translational modifications or gene promotion can be likewise represented, as long as their role has immediate effects on the ability of a target to perform its task.

As ANIMO is a Cytoscape app, models are defined through the Cytoscape user interface (see Fig. 2), where the user inserts a node for each molecular species and an edge for each reaction, with $\rightarrow$ indicating activation and $\dashv$ indicating inhibition similarly to traditional representations of signaling networks (Fig. 1a).

We consider a molecular species (also called reactant) to include all the molecules of the same substance in both their active and inactive state inside the cell. In order to distinguish between the two activity states in which each molecule can be, we define the activity level to represent the percentage of active molecules over an entire molecular species. In an ANIMO model, this value is discretized on a given integer interval. The user can choose the granularity for each molecular species separately, on a scale between 2 (the reactant is seen as either completely inactive or completely active) and 101 levels (allowing to represent activity as $0,1 \%, 2 \% \ldots 100 \%)$. The activity level of a molecular species is 


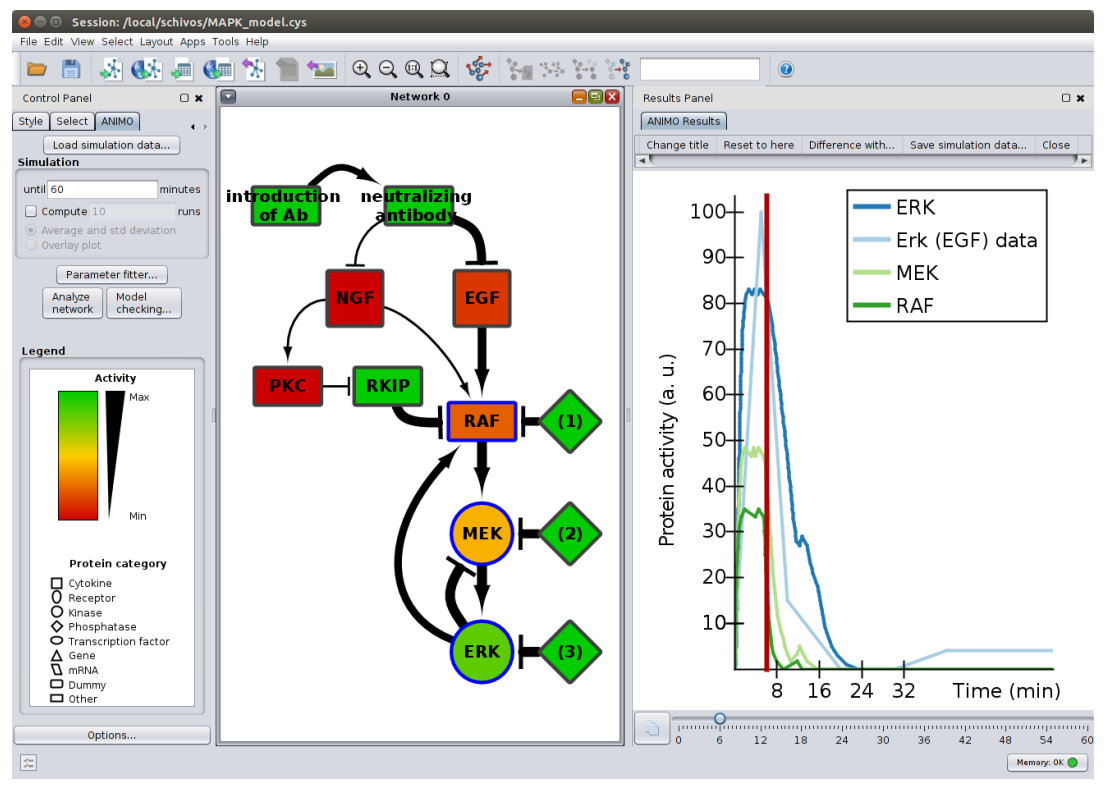

Fig. 2. The Cytoscape 3 user interface running the new ANIMO app (model from [19]). The Network panel in the center contains the nodes-edges model of the cross-talking signaling pathways of growth factors NGF and EGF, with colors indicating node activity levels and shapes representing different protein categories (see the Legend on the left). The Results Panel on the right contains a graph plotting activity levels of selected nodes during the first hour of evolution of the model. The slider under the graph allows the user to select the time instant (marked as a vertical red line in the graph) on which the colors of the nodes in the Network are based. The edge thickness is used to give an idea of the reactions' speed at the selected time instant. The series Erk (EGF) data in the graph is the experimental data from [16] for the $100 \mathrm{ng} / \mathrm{ml}$ EGF treatment.

represented in the ANIMO network by coloring the corresponding node according to the scale shown in the Activity legend in Figure 2, where the minimum indicates that all molecules of the given species are inactive.

The occurrence of a reaction modifies by one discrete step the activity level of its target reactant, making it increase or decrease depending on whether the reaction is defined, respectively, as activating or inhibiting. The rate with which a reaction occurs depends on a formula selected by the user. Choosing one of three available scenarios allows the user to make the reaction rate depend on the activity of one or two reactants. The rate of each reaction can be scaled by modifying the value of one kinetic constant $k$, possibly using a qualitative measure from a predefined set (very slow, slow, medium, fast, very fast). The approximation allows us to reduce the dependence of a model from often unavailable quantitative parameters for biochemical reaction kinetics, while keeping a precision level that is still high enough to be useful. For a more precise explanation on how reaction rates are computed in ANIMO, we recommend [19], where the previously used TA model is presented. The reader interested in the current methods for parameter setting in ANIMO can refer to [17]. 


\section{A new way of modeling signaling pathways with TA}

We present here a novel model to represent signaling pathways with TA in ANIMO. We define the model previously used in ANIMO to be reaction-centered, as for each reaction in the network an instance of a TA template is generated to mimic the occurrences of that reaction. Observing that signaling networks tend to be highly connected, containing noticeably more reactions than reactants, we shift the focus on reactants instead, achieving what we call a reactant-centered model. This change of view is inspired by the classical way in which biological events are modeled with ordinary differential equations (ODEs) [5].

\subsection{The reactant-centered approach}

The reactant-centered model presented here is based on the concept of net effect of a set of reactions on a reactant: instead of considering each reaction in isolation, we consider their combined influence on each reactant. As an example, consider a reactant $A$ activated by reactions $R_{1}$ and $R_{2}$, and inhibited by reaction $R_{3}$ (see Fig. 3a). The net effect of these three reactions on $A$ defines the net reaction $R_{A}=R_{1}+R_{2}-R_{3}$. Applying a concept similar to the definition of an ODE, where the rate of change of each reactant depends on the rate of the reactions influencing it, the rate of $R_{A}$ is computed as the sum of the rates of the reactions influencing $A$ :

$$
r_{A}=r_{1}+r_{2}-r_{3}
$$

where $r_{i}$ is the rate of reaction $R_{i}$ and is defined as follows. Consider $R_{1}$ to be the reaction $B \rightarrow A$ with kinetic constant $k_{1}$. Suppose the settings in the ANIMO network for $R_{1}$ make its rate depend only on the activity level of $B$. Then we compute the rate of $R_{1}$ as $r_{1}=[B] \times k_{1}$, with $[B]$ the current activity level of $B$.

If $r_{A}$ is positive, the activity level of $A$ will increase; otherwise, $A$ will decrease its activity level. The absolute value of $r_{A}$ determines the speed with which such change happens. The value of the reaction rate is thus translated into a time value to be used as time bound in the TA representing $R_{A}$ (see Fig. 3b) by computing

$$
T_{A}=\frac{1}{\operatorname{abs}\left(r_{A}\right)}
$$

with $\operatorname{abs}\left(r_{A}\right)$ the absolute value of $r_{A}$. In order to represent a natural uncertainty or variability in reaction timing, we relax the exact value of $T_{A}$ by defining bounds of $\pm 5 \%$ (which can be changed by the user):

$$
\mathrm{tL}=T_{A} \times 0.95 \quad \mathrm{tU}=T_{A} \times 1.05
$$

Analogously to the reaction-centered model of [19], we will call th the lower time bound and $\mathrm{tU}$ the upper time bound. So we replace an exact reaction time by an interval of possible reaction times, implicitly assuming a uniform distribution over this interval. 


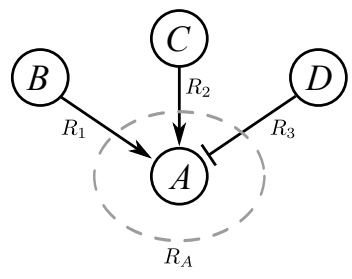

(a)

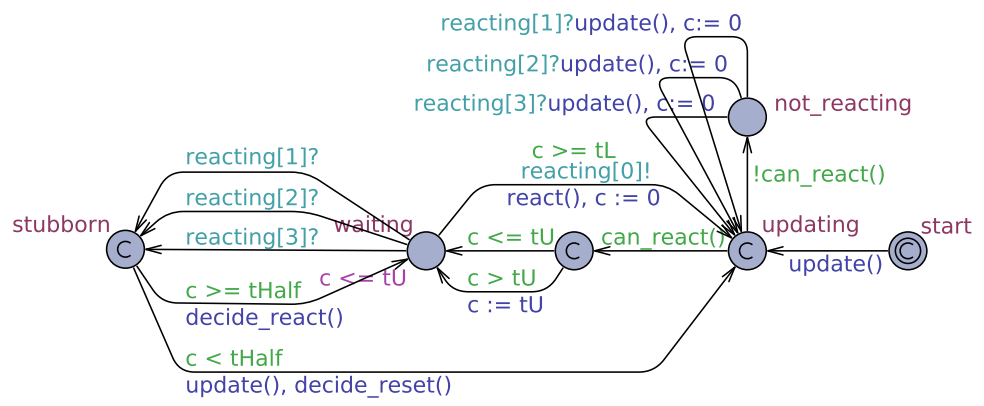

(b)

Fig. 3. (a) A signaling network where one node is influenced by three distinct reactions. The (virtual) reaction $R_{A}$ is defined in the reactant-centered model as the algebraic sum of the reactions influencing $A$. (b) TA template generated by ANIMO for the reactant-centered model of the network in (a). The template has been edited to increase readability. All the functions used in (b) are described in Section 3.2.

\subsection{The new Timed Automata model}

The TA model we propose uses one TA template to represent each reactant: in Figure $3 \mathrm{~b}$ we show the automaton that models how the activity level of reactant $A$ is changed by the (virtual) reaction $R_{A}$. Note that automata for $B, C$ and $D$ are not needed in our example, as no reactions influence them. We will now explain how the TA template for $R_{A}$ works and how its discrete behavior approximates a continuous model.

The proposed TA template fulfils two main tasks:

- perform the reaction, changing the activity level of the modelled reactant $A$

- update the time bounds $\mathrm{tL}$ and $\mathrm{tU}$ in response to changes in the network

The occurrence of the reaction is modelled with the central location waiting, where the automaton waits for the internal clock $c$ to reach the time interval $[\mathrm{tL}, \mathrm{tU}]$; when the condition $\mathrm{tL} \leq c \leq \mathrm{tU}$ is verified, the reaction can occur. This takes the automaton to location updating and simultaneously changes the activity level of $A$ (react() in the transition). Together with all the remaining locations, updating is used to update the time bounds $\mathrm{tL}$ and $\mathrm{tU}$ to reflect the new state of the network and to determine how the reaction $R_{A}$ will continue. 
Note that all locations apart from waiting and not_reacting are declared as committed ( $\mathrm{C}$ inside the location's circle): we use them to perform instant updates to the automaton and its variables. In UPPAAL, no clock advances as long as an automaton is in a committed location, and transitions exiting from committed locations have the precedence over other transitions.

Location updating: decide whether $\boldsymbol{A}$ can change. All transitions entering location updating call the function update() (a call to update() is performed inside react() before returning), which performs the computations described in Section 3.1 and thus determines the new values of $r_{A}$, tL and $\mathrm{tU}$. At this point, one of the following conditions is true:

1. the newly computed reaction rate $r_{A}$ is 0

2. $A$ has reached its maximum activity level and $r_{A}>0$

3. $A$ has reached the activity level 0 and $r_{A}<0$

4. the boundaries for $A$ 's activity level have not been reached and $r_{A} \neq 0$

In the first three cases, the function can_react() will return false, meaning that the activity level of $A$ cannot be updated further in the current conditions. This enables the transition to not_reacting, as the guard !can_react() is true.

In the case 4 , the reaction $R_{A}$ can still occur (can_react() evaluates to true), so the automaton returns to the waiting location to perform a new step of the reaction. The passage through the interposed committed location allows us to ensure that the invariant $\mathrm{c} \leq \mathrm{t} \mathrm{U}$ of location waiting is always respected. In fact, it is possible that $c$ has not been reset upon entering location updating (transition from stubborn, explained later on).

Channel reacting[]: adapt to changes in the environment. When the reaction $R_{A}$ occurs, $A$ is updated to its new activity level (call to react() when exiting from waiting) and a synchronization is performed, using reacting[0]! to indicate that the activity level of $A$ has changed. reacting[] is an array of broadcast channels, each one associated to a reactant in the network: in the example network, the channel with index 0 corresponds to $A, 1$ to $B$, and so on. The reception of a communication along one of those channels indicates that the corresponding reactant has changed its activity level. The automaton representing $R_{A}$ can receive communications on the channels associated to the three reactants influencing $A$ (see the transitions exiting from not_reacting and those entering stubborn). Note that in UPPAAL synchronizations along broadcast channels allow output (!) actions to be performed even if no receiver is present. However, whenever an automaton performs an output action, any other automaton currently in a location where the corresponding input (?) action is enabled will necessarily synchronize with the sender. In our example, this means that $A$ is able to perform the output action reacting[0]! even if no other automaton is present in the network, and would be able to react to any changes in $B, C$ or $D$ if automata representing those reactants were added to the model. 
Location stubborn: reduce the approximation error. The transitions entering location stubborn after a synchronization on a reacting[] channel allow to respond to a change in the environment while $R_{A}$ is occurring. In this event, one of the following conditions is true:

1. at least half of the current reaction step has been performed

2. less than half of the current reaction step has been performed

In case 1 ( $\mathrm{c} \geq \mathrm{tHalf}=\frac{T_{A}}{2}$, transition to waiting), the current reaction step will be completed immediately if the effect of the changes occurred in the reactants influencing $A$ is dramatic. We define a change to be dramatic if it causes the new value of $r_{A}$ to be at least twice the old value, or if it changes the direction of the reaction $\left(r_{A}\right.$ changes sign). The comparison between the possible values of $r_{A}$, together with the actions to immediately enable ${ }^{1} R_{A}$, are taken in the function decide_react(). From this behavior comes the name of location stubborn.

In case 2 ( $c<$ tHalf, transition to updating), the new status of the system is immediately acknowledged (call to function update()) without performing the reaction first. Here, a decision is taken on whether to reset clock c, i.e. whether to throw away the "job already done" or not. Again, the decision depends on the change: a dramatic change causes clock $\mathrm{c}$ to be reset, while a non-dramatic change implies that the work will proceed at a slightly different speed instead of being restarted. This allows to avoid starvation for $R_{A}$ in case of a series of changes with minor effects. Note that in some conditions we could have $c>t U$ : this means that with the new configuration the reaction should already have occurred. Thanks to the committed location leading to location waiting we make sure that the reaction occurs as soon as possible without violating the invariant.

Locations not_reacting and start. While the automaton cannot perform any change to $A$ 's activity level, it waits in location not_reacting for changes in the reactants from which $A$ depends. In the event of any such changes, location updating is reached to check on the feasibility of $R_{A}$.

Location start is used to perform the first step of the model, which is to initialize the parameters of the automaton.

\subsection{Computations on the fly}

Reaction rates are all computed at run-time by the function update(), and are based on the user-chosen scenarios, their kinetic constant $k$ and the activity level of the involved reactants. As such computations require floating-point precision but UPPAAL only provides integer variables and operators, we use a significandand-exponent notation with 4 significant figures, which allows for an error in the order of $0.1 \%$ while avoiding integer overflow in UPPAAL's engine. For example, the floating point number $a=1.23456$ will be represented as the pair $\langle 1235,-3\rangle$, which is translated back as $a=1235 \times 10^{-3}=1.235$. The interested

\footnotetext{
${ }^{1}$ In practice, we set $\mathrm{tL}=\mathrm{tU}=\mathrm{c}=0$.
} 
reader can find the UPPAAL definitions and functions needed to compute rate and time values for the TA templates, together with all other functions such as update() and react(), inside any UPPAAL model file generated by ANIMO with a reactant-centered model type ${ }^{2}$.

\section{Reaction-centered VS reactant-centered}

We will now apply some basic model checking queries to the case study presented in [19], measuring the performances of the two modeling approaches. This will allow us to evaluate the benefit brought by the shift in perspective from a reactionto a reactant-centered model.

All experiments were carried out on an Intel® Core ${ }^{\mathrm{TM}}$ i7 $\mathrm{CPU}$ at $2.80 \mathrm{GHz}$ equipped with $12 \mathrm{~Gb}$ RAM and running Ubuntu GNU/Linux 16.04 64bit. UPPAAL version 4.1.19 64bit was used to compute the result of the queries, asking for "some trace" with random depth-first search order when an execution trace was expected to be produced. For the simulation queries using the statistical model checking engine, we left all options at their default values.

The case study we use as a testbed is the network model shown in the Network panel in Figure 2, which represents signaling events downstream of growth factors EGF (epidermal growth factor) and NGF (nerve growth factor) in PC12 cells (a cell line used to study neuronal differentiation). The model topology proposed in [16] was analyzed with an ANIMO model based on the reaction-centered approach, reproducing the experimentally observed ERK (extracellular signalregulated kinase) activity changes [19]. ${ }^{3}$ In particular, a 10 minutes stimulation with EGF resulted in transient behavior (i.e. peak-shaped, see also the graph in Fig. 2), while NGF stimulation led to sustained activity (data not shown).

\subsection{Simulation cost}

We start by evaluating the cost of simulation with the two different models. This is a particularly important aspect to consider, as during the model building phase a user may need to perform a large number of simulations, continuously adapting the topology or quantitative parameters of a network model. In order to make the modeling approach in ANIMO as interactive as possible, it is desirable to decrease waiting times, and this translates into reducing the computational cost of model analysis as much as possible. In this experiment, we query UPPAAL for simulation runs on the models generated by ANIMO applying the reaction- and reactant-centered modeling approaches to the case study. To define the initial state of the model, we consider the starting condition to be the treatment with $50 \mathrm{ng} / \mathrm{ml} \mathrm{NGF}$, which translates into setting the activity level of node NGF to $15 / 15$, while changing EGF to be at $0 / 15$ activity. This configuration was chosen

\footnotetext{
${ }^{2}$ Models generated by ANIMO are saved in the system's temporary directory. Further details are available in the ANIMO manual at http://fmt.cs.utwente.nl/tools/ animo/content/Manual.pdf

${ }^{3}$ Model available at http://fmt.cs.utwente.nl/tools/animo/models.html
} 
as it generates a more interesting behavior from the biological point of view, also w.r.t. the model checking queries in Section 4.2; the treatment with $100 \mathrm{ng} / \mathrm{ml}$ EGF was also tested and gave similar performance results. Table 1 illustrates the computation time and memory usage when performing 100 simulation runs on each of the two considered models. Computing the simulation runs took about 91\% less time with the reactant-centered model, using 97\% less memory. This decrease in computation time for long simulation runs brings the approach nearer to the idea of interactive exploration of a network.

\begin{tabular}{|l||r|r|}
\hline Model type & Time (s) & Memory (peak KB) \\
\hline Reaction-centered & 30.72 & 291576 \\
\hline Reactant-centered & 2.86 & 9768 \\
\hline
\end{tabular}

Table 1. UPPAAL processor time and memory usage for reaction- and reactant-centered modeling approaches when computing the query simulate 100 [36000] $\{\mathrm{R} 1, \mathrm{R} 2, \ldots, \mathrm{R} 11\}$ on the model from [19] with starting condition $\mathrm{NGF}=15 / 15, \mathrm{EGF}=0 / 15$, corresponding to a treatment with 50 $\mathrm{ng} / \mathrm{ml} \mathrm{NGF}$. The query asks for 100 time series of the activity levels of all reactants in the model over the first 60 minutes of execution.

The scalability of the reactant-centered model was further tested performing 100 simulation runs on a much larger network, consisting of 93 nodes and 297 edges $[21,20]$. The network represents the main signaling and transcription events involved in osteoarthritis in human chondrocytes (cells involved in the production and maintainance of cartilage). In the test, we analyzed a particularly complex scenario, which models a possible path from healthy to osteoarthritic chondrocyte. Using the reactant-centered approach required 757.14 seconds of CPU time and $128840 \mathrm{KBs}$ of memory, while the analysis of the reaction-centered did not terminate after several hours.

\subsection{Model checking performances}

Next, we set out to test the model checking performances on the two versions of the TA model, comparing the execution times and memory requirements for a number of interesting queries:

- (1) and (2): A [] not deadlock. The model continues to execute indefinitely (A refers to all possible paths in the transition system of the model, and [] asks the property to always hold along a path).

$-(3):$ RKIP $<10-->$ ERK $>=40$. After RKIP (Raf kinase inhibitory protein) activity has been lowered, ERK activity increases. As in the model RKIP has 20 levels of granularity and ERK has 100 levels, RKIP $<10$ means that RKIP is less than half active, and ERK $>=40$ means that ERK activity is at least $40 \%$.

- (4): E<> RKIP < 10. Find a point when RKIP is low (E asks for the existence of at least one path for which the property holds, while <> requires the property to hold at least once in a given path). This query is expected to 
generate a trace, the last point of which will be used as initial configuration for model checking queries (5) and (6).

- (5): A [] ERK < 70 and (6): A [] ERK > 35. Once RKIP activity has significantly decreased, ERK activity is sustained at an intermediate level.

The initial conditions are:

- (1): $\mathrm{EGF}=15 / 15$ and $\mathrm{NGF}=0 / 15$, all others as the original configuration, corresponding to the treatment condition with $100 \mathrm{ng} / \mathrm{ml}$ EGF.

- (2) - (4): $\mathrm{EGF}=0 / 15, \mathrm{NGF}=15 / 15$, all others as the original configuration, corresponding to the treatment condition with $50 \mathrm{ng} / \mathrm{ml} \mathrm{NGF}^{4}$.

- (5) and (6): all activities as in the last state of the trace computed from query (4).

We note that performing model checking means dealing with state space explosion problems, and model reduction is recommendable in order to obtain any result within adequate time limits. One of the most user-accessible ways available in ANIMO to reduce the size of a model is setting the "natural uncertainty" defined in Sect. 3.1 to 0 before performing model checking queries. In order to still consider some biological variability, the user can manually perform multiple model checking queries with changed interaction parameters. The tests performed in this section have an uncertainty level set to 0 , instead of the $5 \%$ recommended for simulation-based experiments, to make model checking feasible within seconds.

\begin{tabular}{|c|c|c|c|c|c|c|}
\hline \multirow[b]{2}{*}{ Query } & \multicolumn{2}{|c|}{ Reaction-centered } & \multicolumn{2}{|c|}{ Reactant-centered } & \multicolumn{2}{|c|}{ Improvement } \\
\hline & $\begin{array}{l}\text { Computation } \\
\text { time }(\mathrm{s})\end{array}$ & $\begin{array}{c}\text { Memory usage } \\
\text { (peak KB) }\end{array}$ & $\begin{array}{c}\text { Computation } \\
\text { time }(\mathrm{s})\end{array}$ & $\begin{array}{l}\text { Memory usage } \\
\text { (peak KB) }\end{array}$ & $\begin{array}{c}\text { Time } \\
(\mathrm{n}-\text { fold })\end{array}$ & $\begin{array}{l}\text { Memory } \\
\text { (n-fold) }\end{array}$ \\
\hline (1) & 126.56 & 523448 & 1.04 & 9236 & 122 & 57 \\
\hline (2) & 159.29 & 436496 & 1.73 & 11480 & 92 & 38 \\
\hline (3) & 146.09 & 439484 & 1.04 & 10384 & 140 & 42 \\
\hline (4) & 0.74 & 293508 & 0.06 & 7484 & 12 & 39 \\
\hline (5) & 581.79 & 448764 & 6.86 & 16880 & 85 & 27 \\
\hline (6) & 561.01 & 449248 & 6.42 & 15852 & 87 & 28 \\
\hline
\end{tabular}

Table 2. UPPAAL processor time and memory usage for reaction- and reactant-centered modeling approaches when computing the given queries on the case study from [19].

The queries were used with both the reaction- and reactant-centered TA models, and returned the same results as expected: in particular, query (1) returned false and all other queries returned true. From the biological point of view, the answer to query (1) confirms that under EGF treatment no other activity is observed in the model after the initial peak, while query (2) confirms

\footnotetext{
${ }^{4}$ In the laboratory experimental setting, NGF is used at a lower concentration than EGF, but it is still enough to saturate all NGF receptors, which are rarer than EGF receptors.
} 
that with NGF activity continues indefinitely. Moreover, queries (3) - (6) confirm the result of the simulations shown in [19], with NGF treatment leading to sustained ERK activity.

The results of the model checking performance test are shown in Table 2.

\subsection{Analysis of the results}

Requesting a full inspection of the state space as we do when using a query of the type $\mathrm{A}[] \phi$ returning true in cases (5) and (6), allows us to indirectly compare the state space size of the two model versions. As the computation time improvements in Table 2 show, the reactant-centered model produces indeed a noticeably smaller state space, allowing for a higher level of interactivity also when performing non-trivial model checking. Moreover, our experiments point out that the reactant-centered approach considerably lowers the memory requirements for the model. This is not only due to the absence of possibly large precomputed time tables, which can contain thousands of elements each. Indeed, this point was further investigated by implementing a reaction-centered model which avoids the use of tables and instead makes on-the-fly computations of the time bounds with the same number representation as in the reactant-centered model. This resulted in improved performances in the cases of reachability and simulation-based queries, with memory requirements closer to the ones for the reactant-centered model (0.69 s and $24796 \mathrm{~KB}$ for query (4)). However, in all other cases a much larger amount of memory (around $2 \mathrm{~Gb}$ ) was used with respect to the table-based implementation of the same model, without leading to appreciable benefits in terms of execution time: in some cases performances were noticeably deteriorated (600-800 s for queries (1)-(3)). These findings seem to support the idea that reactant-centered models have a smaller state space.

\section{Model checking in ANIMO}

In order to allow a non-expert user to profit from the power of model checking, we have implemented a template-based user interface to define queries directly inside the ANIMO Cytoscape App: Figure 4 shows the interface for composing a model checking query in ANIMO. The mappings between user interface templates and actual model checking queries were inspired by the ones proposed in [15], and are shown in Table 3.

If the answer to a model checking query contains a (counter-) example trace, the trace is automatically parsed by ANIMO and presented to the user in form of a graph of activity levels, in the same fashion as is normally done with simulation runs. Finally, a button positioned near the time slider under a simulation graph allows the user to easily change the initial activity levels of the whole network by setting them as in the currently selected time instant. This feature was used after executing query (4) to set the initial conditions for queries (5)-(6). Such an addition makes it easier to inspect the behavior of a network by using a sequence of model checking interrogations. 


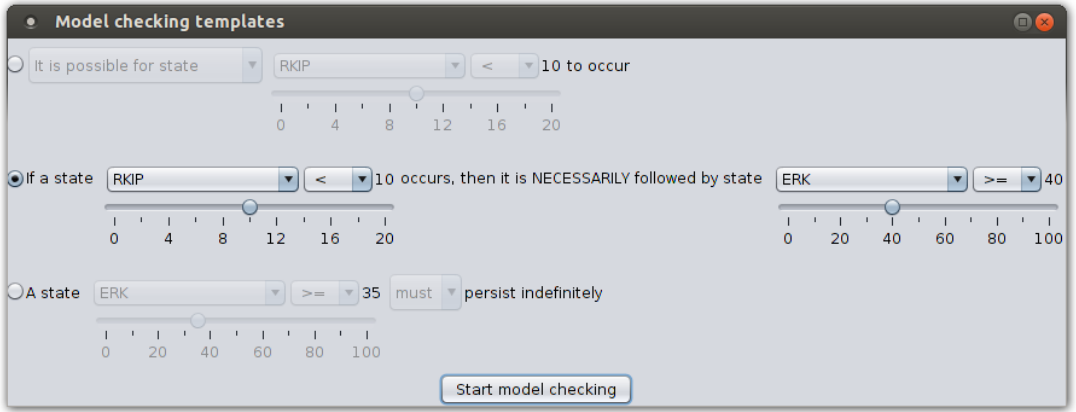

Fig. 4. The interface used in ANIMO to compose a model checking query. The settings on the three lines correspond, from top to bottom, to queries (4), (3) and (6).

\begin{tabular}{|l|l|}
\hline ANIMO template & UPPAAL formula \\
\hline \hline It is possible for state $\phi$ to occur & $\mathrm{E}<>\phi$ \\
\hline State $\phi$ never occurs & $\mathrm{A}[] !(\phi)$ \\
\hline $\begin{array}{l}\text { If a state } \phi \text { occurs, then it is } \\
\text { necessarily followed by a state } \psi\end{array}$ & $\phi-->\psi$ \\
\hline A state $\phi$ can persist indefinitely & $\mathrm{E}[] \phi$ \\
\hline A state $\phi$ must persist indefinitely & $\mathrm{A}[] \phi$ \\
\hline
\end{tabular}

Table 3. Mapping between queries as presented in ANIMO user interface and the corresponding model checking queries in UPPAAL syntax. State formulas indicated by $\phi$ and $\psi$ are all in the form $R \bowtie n$, with $R$ the identifier of a reactant in the model, $\bowtie \in\{<, \leq,=, \geq\rangle$,$\} and n \in[0, g(R)]$ a valid activity level value between 0 and the granularity (number of discrete levels) of $R$.

\section{Conclusions and future work}

We have presented here how the ANIMO tool was improved to provide a more interactive modeling process. Thanks to the increased performances of the new reactant-centered modeling approach, we are able to obtain answers to model checking queries in a matter of seconds. The features of model checking are made accessible without the need to directly deal with TA models. In this way, ANIMO acts as an intermediary between the biologist and a formal representation of biological signaling pathways, letting the experts concentrate on investigating the mechanisms of cellular responses.

In order to enforce the concept of user interaction as a primary focus of the tool, we plan to extend ANIMO with support for parameter sensitivity analysis and parameter fitting, as a follow-up to what was presented in [17]. Moreover, inspired by a work on automata learning [25], we plan to add also the possibility to automatically derive a network topology based on experimental data and previous knowledge.

We aim at widening the available set of model checking queries, in order to allow biologists to perform in silico experiments on an already fitting model and to obtain answers to more relevant questions. This would increase the usefulness of a model as a help to drive wet-lab investigation. In order to allow for 
meaningful in silico experiments, we plan to purposefully introduce user-defined non-deterministic parts in our models, which would allow for drug dosage investigations through model checking. This can be done e.g. through the definition of intervals for the values of some reaction kinetic constants, adding considerable uncertainty in the timing of those reactions. Interesting work has been done on statistical model checking using UPPAAL SMC on models created via ANIMO [7]. Finally, in order to further improve performances, the extension of ANIMO with support for a multi-core model checking approach based on the work by Dalsgaard et al. [6] is under study.

\section{References}

1. R. Alur and D. L. Dill. A theory of timed automata. Theor. Comput. Sci., 126:183235, April 1994.

2. ANIMO. http://fmt.cs.utwente.nl/tools/animo.

3. F. Ciocchetta and J. Hillston. Bio-PEPA: A framework for the modelling and analysis of biological systems. Theor. Comput. Sci., 410:3065-3084, August 2009.

4. Cytoscape 3 ANIMO app. http://apps.cytoscape.org/apps/animo.

5. B. J. Daigle, B. S. Srinivasan, J. A. Flannick, A. F. Novak, and S. Batzoglou. Current progress in static and dynamic modeling of biological networks. In S. Choi, editor, Systems Biology for Signaling Networks, volume 1 of Systems Biology, pages 13-73. Springer New York, 2010.

6. A. E. Dalsgaard, A. W. Laarman, K. G. Larsen, M. C. Olesen, and J. C. van de Pol. Multi-core reachability for timed automata. In M. Jurdzinski and D. Nickovic, editors, 10th International Conference on Formal Modeling and Analysis of Timed Systems, FORMATS 2012, London, UK, volume 7595 of Lecture Notes in Computer Science, pages 91-106, London, September 2012. Springer Verlag.

7. A. David, K. G. Larsen, A. Legay, M. Mikučionis, D. B. Poulsen, and S. Sedwards. Statistical model checking for biological systems. International Journal on Software Tools for Technology Transfer, 17(3):351-367, 2015.

8. H. de Jong, J. Geiselmann, C. Hernandez, and M. Page. Genetic Network Analyzer: qualitative simulation of genetic regulatory networks. Bioinformatics, 19(3):336344, Feb. 2003.

9. L. Dematté, C. Priami, and A. Romanel. Modelling and simulation of biological processes in BlenX. SIGMETRICS Perform. Eval. Rev., 35:32-39, March 2008.

10. P. V. Hornbeck, I. Chabra, J. M. Kornhauser, E. Skrzypek, and B. Zhang. PhosphoSite: A bioinformatics resource dedicated to physiological protein phosphorylation. Proteomics, 4(6):1551-1561, 2004.

11. M. Kanehisa and S. Goto. KEGG: Kyoto Encyclopedia of Genes and Genomes. Nucleic Acids Research, 28(1):27-30, Jan. 2000.

12. S. Killcoyne, G. W. Carter, J. Smith, and J. Boyle. Cytoscape: a communitybased framework for network modeling. Methods in molecular biology (Clifton, N.J.), 563:219-239, 2009.

13. K. G. Larsen, P. Pettersson, and W. Yi. UPPAAL in a nutshell. International Journal on Software Tools for Technology Transfer (STTT), 1:134-152, 1997.

14. P. Mendes, S. Hoops, S. Sahle, R. Gauges, J. Dada, and U. Kummer. Computational modeling of biochemical networks using COPASI systems biology. volume 500 of Methods in Molecular Biology, chapter 2, pages 17-59. Humana Press, Totowa, NJ, 2009. 
15. P. T. Monteiro, D. Ropers, R. Mateescu, A. T. Freitas, and H. de Jong. Temporal logic patterns for querying dynamic models of cellular interaction networks. Bioinformatics, 24(16):i227-i233, 2008.

16. S. D. M. Santos, P. J. Verveer, and P. I. H. Bastiaens. Growth factor-induced MAPK network topology shapes Erk response determining PC-12 cell fate. Nature Cell Biology, 9(3):324-330, Feb. 2007.

17. S. Schivo, J. Scholma, H. B. J. Karperien, J. N. Post, J. C. van de Pol, and R. Langerak. Setting parameters for biological models with ANIMO. In E. André and G. Frehse, editors, Proceedings 1st International Workshop on Synthesis of Continuous Parameters, Grenoble, France, volume 145 of Electronic Proceedings in Theoretical Computer Science, pages 35-47. Open Publishing Association, April 2014.

18. S. Schivo, J. Scholma, P. E. van der Vet, M. Karperien, J. N. Post, J. van de Pol, and R. Langerak. Modelling with ANIMO: between fuzzy logic and differential equations. BMC Systems Biology, 10(1):56, 2016.

19. S. Schivo, J. Scholma, B. Wanders, R. Urquidi Camacho, P. van der Vet, M. Karperien, R. Langerak, J. van de Pol, and J. Post. Modelling biological pathway dynamics with Timed Automata. IEEE Journal of Biomedical and Health Informatics, 18(3):832-839, 2013.

20. J. Scholma, J. Kerkhofs, S. Schivo, R. Langerak, P. E. van der Vet, H. B. J. Karperien, J. C. van de Pol, L. Geris, and J. N. Post. Mathematical modeling of signaling pathways in osteoarthritis. In S. Lohmander, editor, 2013 Osteoarthritis Research Society International (OARSI) World Congress, Philadelphia, USA, volume 21, Supplement, pages S123-S123, Amsterdam, April 2013. Elsevier.

21. J. Scholma, S. Schivo, J. Kerkhofs, R. Langerak, H. B. J. Karperien, J. C. van de Pol, L. Geris, and J. N. Post. ECHO: the executable chondrocyte. In Tissue Engineering $\&$ Regenerative Medicine International Society, European Chapter Meeting, Genova, Italy, volume 8, pages 54-54, Malden, June 2014. Wiley.

22. J. Scholma, S. Schivo, R. Urquidi Camacho, J. van de Pol, M. Karperien, and J. Post. Biological networks 101: Computational modeling for molecular biologists. Gene, 533(1):379-384, 2014.

23. W. Siers, M. Bakker, B. Rubbens, R. Haasjes, J. Brandt, and S. Schivo. webANIMO: Improving the accessibility of ANIMO [version 1; referees: 3 approved with reservations]. F1000Research, 5(1714), 2016.

24. M. Tomita, K. Hashimoto, K. Takahashi, T. S. Shimizu, Y. Matsuzaki, F. Miyoshi, K. Saito, S. Tanida, K. Yugi, J. C. Venter, and C. A. H. III. E-CELL: software environment for whole-cell simulation. Bioinformatics, 15(1):72-84, Jan. 1999.

25. J. Tretmans. Model-based testing and some steps towards test-based modelling. In M. Bernardo and V. Issarny, editors, Formal Methods for Eternal Networked Software Systems, volume 6659 of Lecture Notes in Computer Science, pages 297326. Springer Berlin / Heidelberg, 2011. 